\title{
Ablation of $\mathrm{p} 75^{\mathrm{NTR}}$ signaling strengthens gamma-theta rhythm interaction and counteracts $A \beta$-induced degradation of neuronal dynamics in mouse hippocampus in vitro
}

\author{
Yuniesky Andrade-Talavera (1)', Hugo Balleza-Tapia', Pablo Dolz-Gaitón', Gefei Chen², Jan Johansson² and \\ André Fisahn ${ }^{1}$
}

\begin{abstract}
Gamma and theta brain rhythms play important roles in cognition and their interaction can affect gamma oscillation features. Hippocampal theta oscillations depend on cholinergic and GABAergic input from the medial septumdiagonal band of Broca. These projecting neurons undergo degeneration during aging and maintain high levels of neurotrophin receptor p75 (p75 NTR). p75 NTR mediates both apoptosis and survival and its expression is increased in Alzheimer's disease (AD) patients. Here, we investigate the importance of $p 75^{\mathrm{NTR}}$ for the cholinergic input to the hippocampus. Performing extracellular recordings in brain slices from $\mathrm{p} 75^{\mathrm{NTR}}$ knockout mice $\left(\mathrm{p} 75^{-/-}\right)$in presence of the muscarinic agonist carbachol, we find that gamma oscillation power and rhythmicity are increased compared to wild-type (WT) mice. Furthermore, gamma activity is more phase-locked to the underlying theta rhythm, which renders a stronger coupling of both rhythms. On the cellular level, we find that fast-spiking interneurons (FSNs) fire more synchronized to a preferred gamma phase in $\mathrm{p} 75^{-1-}$ mice. The excitatory input onto FSN is more rhythmic displaying a higher similarity with the concomitant gamma rhythm. Notably, the ablation of $p 75^{\text {NTR }}$ counteracts the $A \beta$-induced degradation of gamma oscillations and its nesting within the underlying theta rhythm. Our results show that the lack of $p 75^{\mathrm{NTR}}$ signaling could promote stronger cholinergic modulation of the hippocampal gamma rhythm, suggesting an involvement of $p 75^{\text {NTR }}$ in the downregulation of cognition-relevant hippocampal network dynamics in pathologies. Moreover, functional data provided here suggest $\mathrm{p} 75^{\mathrm{NTR}}$ as a suitable target in the search for efficacious treatments to counteract the loss of cognitive function observed in amyloid-driven pathologies such as AD.
\end{abstract}

\section{Introduction}

The synchronization of many of the different interconnected neuronal assemblies present in the

\footnotetext{
Correspondence: Yuniesky Andrade-Talavera (yuniesky.andrade-talavera@ki.se) or André Fisahn (andre.fisahn@ki.se)

${ }^{1}$ Neuronal Oscillations Laboratory, Division of Neurogeriatrics, Department of Neurobiology, Care Sciences and Society, Center for Alzheimer Research, Karolinska Institutet, 17164 Solna, Sweden

${ }^{2}$ Division of Neurogeriatrics, Department of Neurobiology, Care Sciences and Society, Center for Alzheimer Research, Karolinska Institutet, 14183 Huddinge, Sweden

These authors contributed equally: Yuniesky Andrade-Talavera,

Hugo Balleza-Tapia
} 
rhythms that leads to precise neuronal firing patterns. The most common interaction that has been reported is the coupling between theta phase and gamma power ${ }^{4-10}$. Theta-phase to gamma-phase $\mathrm{e}^{11,12}$ and theta power to gamma power couplings have also been described ${ }^{12}$.

Some physiological advantages of theta-gamma coupling are the possibility of encoding different information in different theta phases and the synchronization of neuronal ensembles over long distances ${ }^{13,14}$. It is noteworthy that in addition to modulating gamma oscillations, the theta rhythm can also induce them ${ }^{15}$.

Hippocampal theta rhythm depends partially on the cholinergic and GABAergic input from the medial septum and diagonal band of Broca (MS/DBB) located in the basal forebrain, which has been classically considered as the "pacemaker" for the hippocampal theta activity ${ }^{16}$. Cholinergic fibers are known to innervate both pyramidal cells and interneurons in the hippocampus. Selective septo-hippocampal cholinergic lesions sparing GABAergic inputs can affect the amplitude of theta oscillations $^{17}$. Thus, any factors that influence the development and/or survival of basal forebrain cholinergic neurons (BFCNs) are likely to have a profound impact on the dynamics of cognition-relevant oscillatory behavior in the hippocampus.

Cholinergic neurons of the MS/DBB are known to undergo a moderate degeneration during aging resulting in a decrease of cholinergic function that has been related to the progressive memory deficits during aging ${ }^{18,19}$. This age-dependent degeneration of BFCN might be due to a decrease in trophic support, as in aging an attenuation of the neurotrophic signaling in sensory neurons has been observed $^{20}$. In hippocampal slices, it has been observed that selective ablation of the neurotrophin receptor TrkB in fast-spiking interneurons (FSNs) decreases the amplitude of carbachol (CCh)-induced gamma oscillations ${ }^{21}$. However, much less is known about how neurotrophic signaling may regulate neuronal network rhythms and paramount neuronal populations such as FSNs.

On the other hand, neurotrophins are also known to affect the viability, differentiation, and size of cholinergic neurons ${ }^{22-24}$. The neurotrophin receptor p75 $\left(\mathrm{p} 75^{\mathrm{NTR}}\right)$ has been reported to mediate both proapoptotic signaling and survival, as well as neurite growth ${ }^{25}$. BFCNs are among the few neuronal cell types that maintain p $75^{\mathrm{NTR}}$ expression at high levels during adulthood. Van der Zee et al. ${ }^{26}$ first reported an increased number of cholinergic neurons in the septum of mice carrying a targeted mutation in the third exon of the $p 75^{N T R}$ gene. Conversely, $\mathrm{p} 75^{\mathrm{NTR}}$ overexpression significantly reduces dendritic complexity as well as spine density in CA1 pyramidal cells ${ }^{27}$. In addition, p $75^{\mathrm{NTR}}$ interacts directly with amyloid-beta peptide $(A \beta)$ mediating neuritic dystrophy $^{28,29}$.
Such interaction points toward the deleterious effect of the presence of $\mathrm{p} 75^{\mathrm{NTR}}$ in age-related neurodegenerative diseases like Alzheimer's disease (AD). Although p $75^{\text {NTR }}$ has an evident effect on the overall cholinergic function in the brain, particularly on the survival and innervation of $\mathrm{BFCN}$ having the potential to modulate both hippocampal theta and gamma oscillations, the role of $\mathrm{p} 75^{\mathrm{NTR}}$ on these oscillatory rhythms in normal and pathological conditions remains unknown. As such, it has been observed that $A \beta$ induces dysfunction of glutamatergic neurons impairing septum rhythmicity. This may negatively affect hippocampal rhythmogenesis and underlie the memory loss observed in $\mathrm{AD}^{30}$.

Here, we describe the effect of p $75^{\text {NTR }}$ ablation of increasing cognition-relevant hippocampal rhythms such as gamma oscillations, elucidate the underlying cellular mechanisms in FSN, and how it strengthens the interaction with the underlying theta oscillations in mouse brain slices. We show that lack of $\mathrm{p} 75^{\mathrm{NTR}}$ signaling protects the hippocampal network against pathophysiological changes observed in $\mathrm{AD}$ such as the $\mathrm{A} \beta$-induced degradation of gamma oscillations and gamma-theta interaction. It suggests $\mathrm{p} 75^{\mathrm{NTR}}$ signaling inhibition as a potential focus for novel therapies against $\mathrm{AD}$ including cognitiverelevant rhythms strengthening.

\section{Methods \\ Animals}

Experiments were performed in accordance with the ethical permit granted by Norra Stockholms Djurförsöksetiska Nämnd to AF (N45/13). p75 ExonIII+/- mice $\left(\mathrm{p} 75^{+/-}\right.$) with a C57BL/6J background were purchased from Jackson Laboratory (USA). Animals were inbred in order to obtain the $\mathrm{p} 75^{+/+}$and $\mathrm{p} 75^{-/-}$mice used for experiments. The number of animals was reduced to a minimum by optimizing the use of slices from both hemispheres. The total number of animals was set to a minimum of 3 and a maximum of 5 per group and condition. Male animals between P15 and P21 were deeply anesthetized using isofluorane before being sacrificed by decapitation and brain removal.

\section{Genotyping}

Genotypic analysis was performed by PCR using primer $p 75 \mathrm{com}$ (5'-GGACAAACAGAACACAGTGTGTGA-3') in exon III upstream of the insertion site, and primer $p 75 w t\left(5^{\prime}\right.$-ACCCATATAATCGCTGAGAGAGGA- $\left.{ }^{\prime}\right)$ in the following intron for the detection of the wild-type allele (437 bp amplicon, Fig. S1) and primer $p 75 k o$ (5'GAACTTCCTGACTAGGGGAGGAGT-3') in the PGK promoter of the vector inserted ${ }^{26}$ for the detection of knockout allele (252 bp amplicon, Fig. S1). Cycle parameters were $94{ }^{\circ} \mathrm{C}$ for $4 \mathrm{~min}, 95^{\circ} \mathrm{C}$ for $20 \mathrm{~s}, 60^{\circ} \mathrm{C}$ for $20 \mathrm{~s}$, $72{ }^{\circ} \mathrm{C}$ for $32 \mathrm{~s}, 40$ cycles, and $72^{\circ} \mathrm{C}$ for $7 \mathrm{~min}$. 


\section{Hippocampal slice preparation}

Hippocampal slices were prepared as previously described $^{31}$. Briefly, the brain was dissected out and placed in ice-cold artificial cerebrospinal fluid (ACSF) modified for dissection containing (in $\mathrm{mM}$ ) $80 \mathrm{NaCl}, 24$ $\mathrm{NaHCO}_{3}, 25$ glucose, $1.25 \mathrm{NaH}_{2} \mathrm{PO}_{4}, 1$ ascorbic acid, 3 Na pyruvate, $2.5 \mathrm{KCl}, 4 \mathrm{MgCl}_{2}, 0.5 \mathrm{CaCl}_{2}, 75$ sucrose, and bubbled with carbogen $\left(95 \% \mathrm{O}_{2}\right.$ and $\left.5 \% \mathrm{CO} 2\right)$. Horizontal sections ( $350 \mu \mathrm{m}$ thick) of the ventral hippocampi of both hemispheres were prepared with a Leica VT1200S vibratome (Leica Microsystems). After cutting, slices were transferred into a humidified interface holding chamber containing standard ACSF (in $\mathrm{mM}$ ): $124 \mathrm{NaCl}, 30$ $\mathrm{NaHCO}_{3}, 10$ glucose, $1.25 \mathrm{NaH}_{2} \mathrm{PO}_{4}, 3.5 \mathrm{KCl}, 1.5 \mathrm{MgCl}_{2}$, and $1.5 \mathrm{CaCl}_{2}$, continuously supplied with humidified carbogen gas $\left(5 \% \mathrm{CO}_{2}, 95 \% \mathrm{O}_{2}\right)$. The chamber was held at $37^{\circ} \mathrm{C}$ during slicing and subsequently allowed to cool down to room temperature for at least $1 \mathrm{~h}$ before the commencement of experiments.

\section{Electrophysiology}

For local field potential (LFP) recordings glass microelectrodes (4-6 M $\Omega$ ) filled with standard ACSF were placed in CA3 stratum pyramidale. Single-cell recordings were carried out in a submerged recording chamber. Action potentials (APs) and excitatory postsynaptic potentials (EPSCs) from FSNs in area CA3 were recorded in whole-cell patch-clamp mode with an internal recording solution containing (in $\mathrm{mM}$ ): $122.5 \mathrm{~K}^{+}$gluconate, $8 \mathrm{KCl}, 4 \mathrm{Mg}^{2+}$ ATP, $0.3 \mathrm{Na}^{+}$GTP, 10 HEPES, 0.2 EGTA, $2 \mathrm{MgCl}_{2}$ with $\mathrm{pH}$ set to $7.2-7.3$, and osmolarity to 270-280 mosmol/l. AP firing was recorded in current clamp at free membrane potential.

FSNs were visualized under an upright microscope using infrared-differential interference contrast (IR-DIC) microscopy (Axioskop, Carl Zeis AG, Göttingen, Germany) and were identified and distinguished from other interneuron populations based on their morphology and characteristic firing behavior (Fig. S2) ${ }^{32,33}$ and location in the CA3 area. Interface chamber LFP recordings were performed with a 4-channel M102 amplifier (University of Cologne, Germany). Data were sampled at $5 \mathrm{kHz}$, conditioned using a HumBug $50 \mathrm{~Hz}$ noise eliminator (Quest Scientific), low-pass filtered at $1 \mathrm{kHz}$, digitized (Digidata 1440A, Molecular Devices, CA, USA), and stored on a hard drive using pCLAMP 9.6 software (Molecular Devices). Concomitant LFP and patch-clamp recordings in the submerged-type recording chamber were performed with a patch-clamp amplifier (Multiclamp 700B), and data were acquired using pCLAMP 10.4 software (Molecular Devices). LFP recordings were also conditioned using a HumBug $50 \mathrm{~Hz}$ noise eliminator (Quest Scientific). All signals recorded in submerged configuration were low-pass filtered at $1 \mathrm{kHz}$, acquired at
$5 \mathrm{kHz}$, and digitized and stored using Digidata 1322A and pCLAMP 10.4 software (Molecular Devices, CA, USA).

In all experiments, gamma oscillations were elicited by applying CCh $(20 \mu \mathrm{M})$ to the extracellular bath ${ }^{34}$. Oscillations were allowed to stabilize for at least $20 \mathrm{~min}$ before recording.

\section{Data analysis}

Fast Fourier transformations for power spectra were calculated from 60-s-long LFP data traces (segments of 8192 points) using Axograph X software (Kagi, Berkeley, CA, USA). Gamma oscillation power was calculated by integrating the power spectral density from 20 to $80 \mathrm{~Hz}$ using KaleidaGraph. Theta oscillation power was calculated on $4-10 \mathrm{~Hz}$ filtered traces. Gamma envelope $\left(\gamma_{\mathrm{ENV}}\right.$, see below) power was calculated as the integrated power between 5 and $10 \mathrm{~Hz}$. Peak frequency for each signal was obtained as the frequency at which the maximum power of the respective frequency band was located. Once differences in the peak frequency were detected, further confirmation was derived from analyzing the lag to the second peak in the corresponding autocorrelogram (see below). For further analysis of LFP recordings, the signals were preprocessed using a bandpass Butterworth filter (in both directions to prevent a shift in the phase angle) set to $20-60 \mathrm{~Hz}$ for autocorrelation analysis of gamma oscillations and $4-10 \mathrm{~Hz}$ (theta) for gamma and theta interaction. Normalized autocorrelations were performed using Matlab customwritten routines. The coefficient of rhythmicity $(\mathrm{Cr})$ was calculated from the autocorrelograms as a measure of the quality of gamma and theta oscillations. It was defined as $\mathrm{Cr}=(\alpha-\beta) /(\alpha+\beta)$ including a modification to Andersson et al. ${ }^{35}$ : prior to Cr calculation, $(1+\alpha)$ and $(1+\beta)$ corrections were applied with $\alpha$ corresponding to the value of the height of the second peak and $\beta$ to the first trough in the autocorrelogram (counting the first peak at zero lag). $\mathrm{Cr}$ ranges between 0 and 1 with higher coefficient values denoting more rhythmic activity. Only recordings having a $\mathrm{Cr} \geq 0.01$ were considered rhythmic and included in the study ${ }^{36}$.

A gamma amplitude time series were obtained using a Matlab custom-written routine, where the built-in envelope function $\left(\gamma_{\mathrm{ENV}}\right)$ was set to "peak" mode and peak separation was set to 100 in order to finely adjust the envelope to the amplitude of the signal. In order to assess the reliability of the method, we have analyzed ten recordings from the control group $\left(\mathrm{p} 75^{+/+}\right)$taken at random using different peak adjustment settings (Fig. S3). For the time domain (cross-correlation (XC)) analysis, the filtered theta oscillations $(\theta)$ and the $\gamma_{\mathrm{ENV}}$ were used. Normalized XC between $\theta$ and the $\gamma_{\mathrm{ENV}}\left(\mathrm{XC}\left(\theta, \gamma_{\mathrm{ENV}}\right)\right)$ was performed with a Matlab custom-written routine. The oscillatory nature of the signals was evidenced in the XC 
analysis that displayed several peaks with periodic fluctuations. To describe the similarity and phase shift of both signals, we considered the magnitude and lag of the central negative peak, respectively ${ }^{37}$.

Spike-phase coupling analysis was performed on concomitant LFP recordings and single-cell recordings using a custom-made routine in Matlab to relate the FSN spiking activity to ongoing gamma oscillations ${ }^{32,33,36}$. The 20-40 Hz filtered LFP traces were used and APs were detected using an amplitude threshold. The instantaneous phase angle of gamma oscillations at which an AP occurred was determined by using a Hilbert transform. Phase angles of all AP and gamma oscillation phases were represented in polar plots and expressed in radians with the peak of the oscillation cycle corresponding to $0 \pi$ and the trough to $\pm \pi$ in the polar plots. Each AP was assigned a vector of length 1 with an angle corresponding to the phase of the field at the same time. An averaged resultant phase-density vector was used to describe the preferred phase of firing (phase angle) and how recurrent the firing in that angle was (vector length). Thus, a larger vector denotes a more synchronized AP firing. The vector length is shown normalized by the total number of AP for each cell recorded from both $\mathrm{p} 75^{+/+}$and $\mathrm{p} 75^{-/-}$. All distributions were tested for uniformity. Inclusion criteria were set at $p<0.05$ value by performing a Rayleigh's test.

\section{Drugs and chemicals}

All chemical compounds used in intracellular and extracellular solutions were obtained from Sigma-Aldrich Sweden AB (Stockholm, Sweden). CCh was dissolved in milliQ water.

Met- $A \beta$ residues $1-42$ (referred to as $A \beta$ herein) were produced as previously described ${ }^{38}$. Briefly, $A \beta$ was expressed in BL21*(DE3) pLysS Escherichia coli and purified with DEAE-Sepharose (GE Healthcare). To get rid of large aggregates, the peptides were passed through a $30,000 \mathrm{Da}$ Vivaspin concentrator (GE Healthcare) at $4{ }^{\circ} \mathrm{C}$. The filtrate was concentrated at $4^{\circ} \mathrm{C}$ with a $5000 \mathrm{Da}$ Vivaspin concentrator (GE Healthcare) until $A \beta$ concentration was $\sim 50 \mu \mathrm{M}$. The peptides were aliquoted in low-bind Eppendorf tubes (Axygen) and stored at $-20^{\circ} \mathrm{C}$.

\section{Statistical analysis}

All statistical analysis was performed using GraphPad Prism. Results are reported as mean \pm SEM. Prior statistical analysis all the data were subjected to an outliers determination and removal with the ROUT (robust regression and outlier removal) method, followed by tests for normality distribution and variance similarity between groups. Due to previous experience with outliers and overall sample behavior, sample size was determined based on previous studies performed in interface-type chambers $^{31,36,38-40}$ as well as in submerged-type chambers $^{31,33,36}$. Tests for statistical significance were performed on absolute values in all the experiments using two- or one-sided when appropriate. Student's $t$ test or Mann-Whitney $U$ test were performed depending on the parametric or nonparametric distribution, respectively. Significance levels were set as follows: ${ }^{*} p<0.05,{ }^{* *} p<0.01$, $* * * 0.005$, and ${ }^{* * * * * *} p<0.0001$.

\section{Results}

Ablation of $\mathrm{p} 75^{\mathrm{NTR}}$ signaling increases gamma oscillation power and rhythmicity as well as gamma-theta phase locking in the hippocampal network

To study the effect of $\mathrm{p} 75^{\mathrm{NTR}}$ signaling loss on cholinergic receptor-driven oscillatory rhythms in the murine hippocampus, we performed LFP recordings in the CA3 region of horizontal hippocampal slices. LFP gamma oscillations were elicited by applying $20 \mu \mathrm{M}$ CCh to the bath solution and allowed to stabilize for no $<20 \mathrm{~min}$. CCh has been shown previously to be able to induce both gamma $(30-80 \mathrm{~Hz})$ and theta $(5-10 \mathrm{~Hz})$ oscillations in vitro ${ }^{34,41-44}$. In our experiments, we were able to record large and consistent gamma oscillations (Fig. 1A, top traces). Theta oscillations were apparent after filtering the LFP signal (Fig. 1A, bottom).

We found that gamma power was significantly increased in $\mathrm{p} 75^{-/-}$mice compared to $\mathrm{p} 75^{+/+}$mice (Fig. 1A, B, D). Interestingly, we also observed a significant leftward shift in the peak frequency of the $\mathrm{p} 75^{-1-}$ power spectra (Fig. 1B, E), suggesting that gamma oscillations in these mice are slower. This finding was further confirmed by performing a gamma oscillation autocorrelation, which showed a shift of the $\mathrm{p} 75^{-/-}$, compared to gamma $\mathrm{p} 75^{+/+}$-gamma signal (Fig. 1B). Gamma autocorrelation analysis showed that $\mathrm{p} 75^{-1-}$ mice display a more rhythmic gamma oscillation compared to $\mathrm{p} 75^{+/+}$ littermates, which is evident from the greater peak-totrough distance (Fig. 1B). The increased gamma rhythmicity was confirmed when calculating the coefficient of rhythmicity $(\mathrm{Cr})$, which was significantly increased for gamma oscillations induced in $\mathrm{p} 75^{-/-}$hippocampal slices (Fig. 1F). No differences in theta power, peak frequency, or $\mathrm{Cr}$ were found between $\mathrm{p} 75^{+/+}$and $\mathrm{p} 75^{-1-}$ mice (Fig. 1A, C-F).

Given that $\mathrm{p} 75^{-1-}$ mice display slower gamma oscillations, we considered the possibility that this could have an effect on the phase-phase modulation between the gamma and theta rhythms. We observed that gamma peaks from $\mathrm{p} 75^{+/+}$were more uniformly distributed around the theta cycle compared to $\mathrm{p} 75^{-1-}$ (Fig. S4A-D). Interestingly, $\mathrm{p} 75^{-1-}$ mice appear to have a narrower fit for a Gaussian-like distribution evidenced by a significant increase of the resultant vector of gamma-peak distribution (Fig. S4A-E). Distributions were also different with regard to the gamma-peak-preferred theta phase angle 


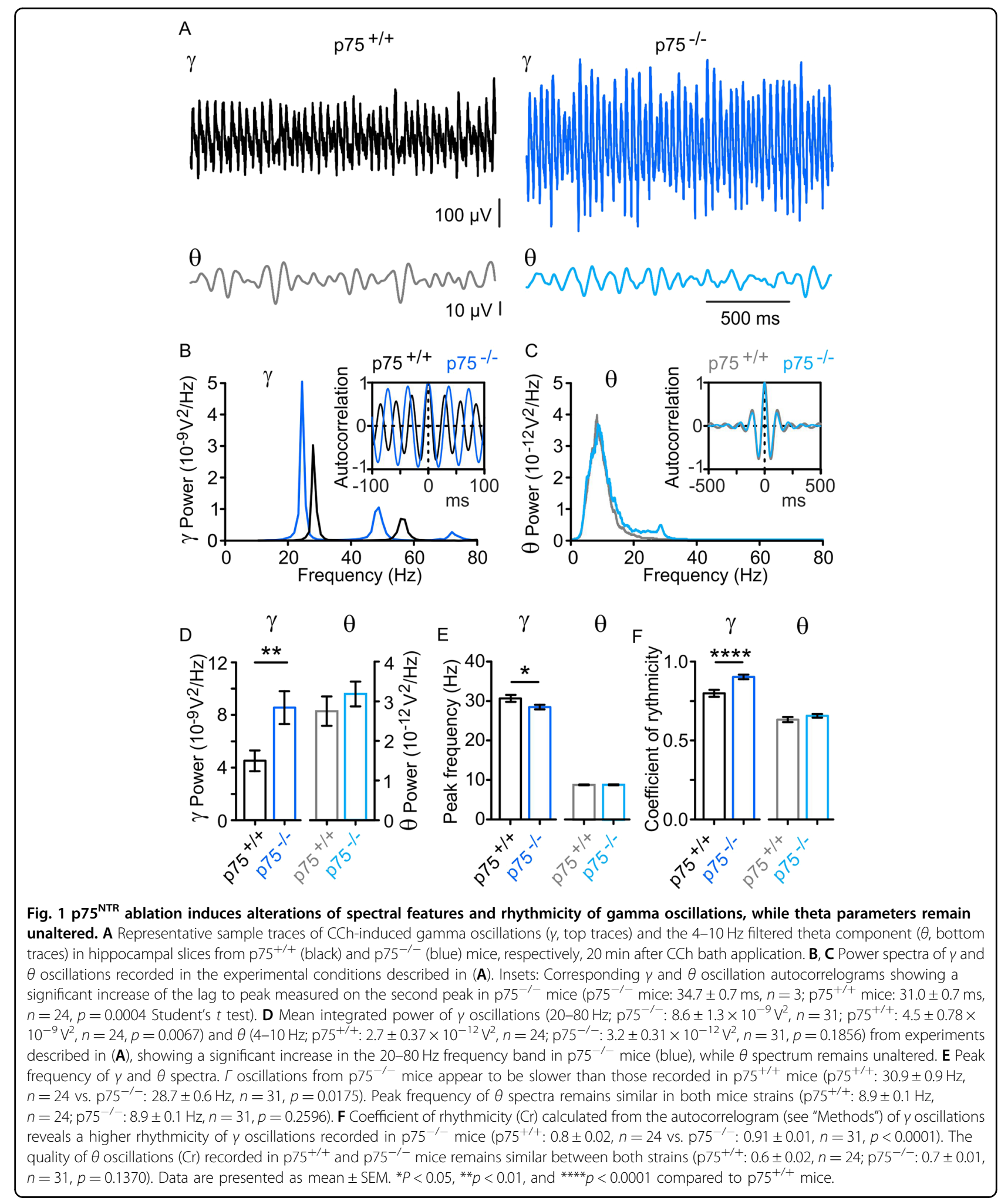

(Fig. S4F). Slower gamma in $\mathrm{p} 75^{-1-}$ mice was further confirmed when calculating the average number of gamma peaks per theta cycle in both mouse strains (Fig. S4A, C, G).

\section{Ablation of $\mathrm{p} 75^{\mathrm{NTR}}$ strengthens gamma-theta nesting}

It has been reported previously that gamma rhythm amplitude can wax and wane in the theta range ${ }^{37}$. Although the theta power obtained after filtering the raw 

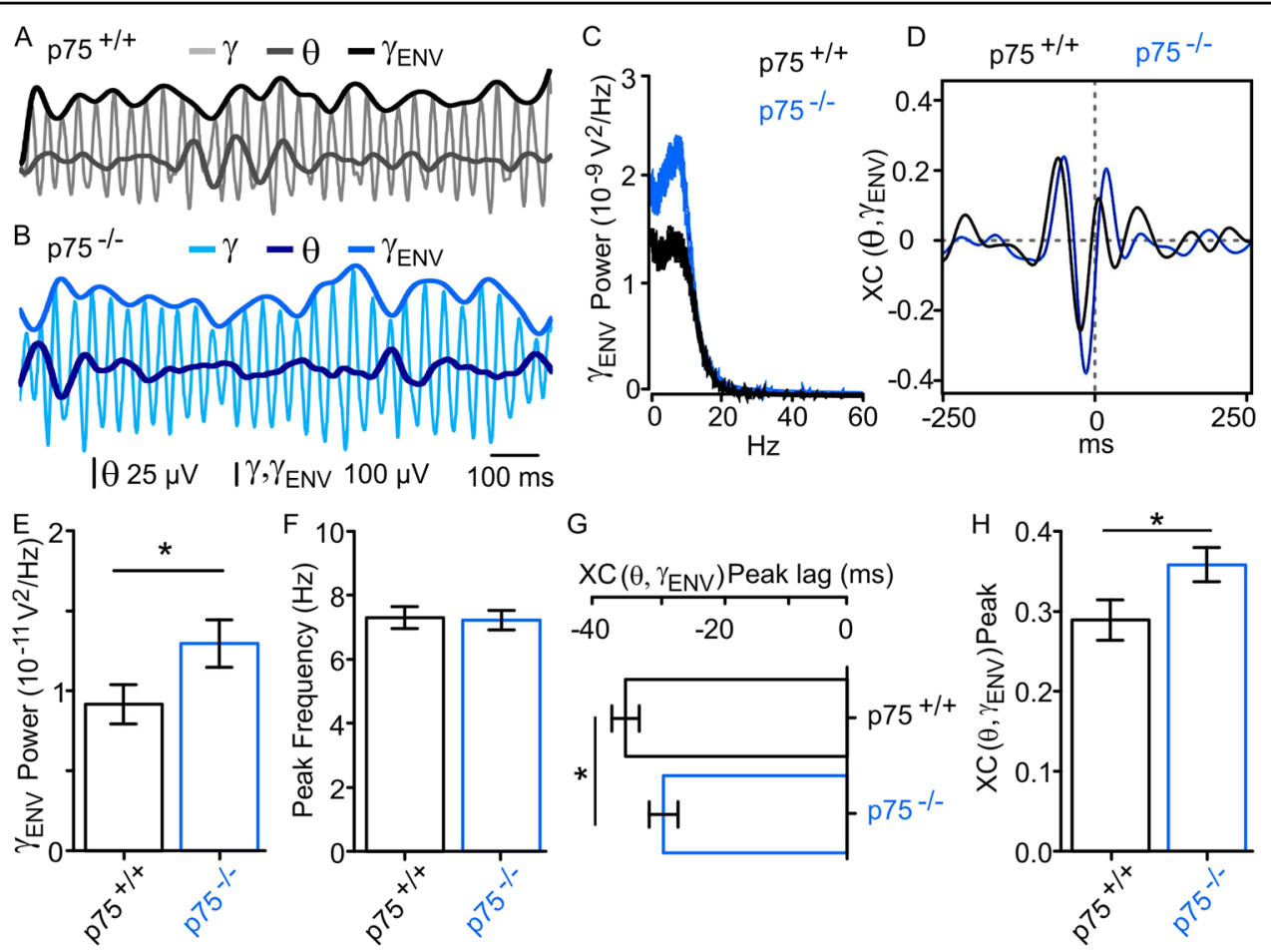

Fig. 2 p75 ${ }^{\mathrm{NTR}}$ ablation strengthens gamma nesting within the theta rhythm. A, B Representative traces of CCh-induced LFPs in the same experimental conditions as in Fig. 1 showing gamma $(\gamma)$ and underlying theta $(\theta)$ oscillations. An envelope signal was adjusted to the amplitude of each $\gamma$ peak ( $\gamma_{\text {ENV }}$, see "Methods") on recordings from p $75^{+/+}$( $\gamma_{\text {ENV: }}$ top black trace; $\gamma$ : gray; $\theta$ : dark gray) and p75 ${ }^{-1-}$ ( $\gamma_{\text {ENV: }}$ top blue trace; $\gamma$ : blue; $\theta$ : dark blue) mice. C Representative power spectra of the $\gamma_{\mathrm{ENV}}$ Oscillation of $\mathrm{p} 75^{+/+}$(black) and $\mathrm{p} 75^{-1-}$ (blue), showing that this oscillation falls within the frequency range of $\theta$ rhythm $(5-10 \mathrm{~Hz})$ in both mouse strains with similar peak frequency and increased power in p75 ${ }^{-1-}$ mice. D Crosscorrelation function of $\theta$ with $\gamma_{\mathrm{ENV}}\left(X C\left(\theta, \gamma_{\mathrm{ENV}}\right)\right)$ obtained from the same data as in $(\mathbf{A}, \mathbf{B})$ reflects the coincidence of $\gamma$ bouts within the $\theta$ range with the trough of $\theta$ as a central prominent negative peak close to zero. The slight lag shift of $X C\left(\theta, \gamma_{\mathrm{ENV}}\right)$ peak in p $75^{+/+}$(black) compared to p75 ${ }^{-/-}$(blue) mice reveals the difference of $\theta-\gamma_{\text {ENV }}$ phase locking quantified in (F) similar to that observed in $\gamma-\theta$ phase locking (refer to Fig. S4). E Power of $\gamma_{\text {ENV }}$ in the $\theta$ frequency band shows a significant increase in the amplitude modulation of $p 75^{-/-}$-gamma oscillations $\left(p 75^{+/+}: 9.2 \pm 1.2 \times 10^{-11} \mathrm{~V}^{2}\right.$, $n=24$ vs. $\left.p 75^{-1-}: 13 \pm 1.5 \times 10^{-11} \mathrm{~V}^{2}, n=31, p=0.0429\right)$. $\mathbf{F}$ Peak frequency of the spectra as in (C) from all slices recorded showing that the amplitude modulation of $\gamma$ is oscillating within the $\theta$ range in both mice strains $\left(p 75^{+/+}: 7.3 \pm 0.3 \mathrm{~Hz}, n=24 \mathrm{vs}\right.$. $p 75^{-1-}: 7.2 \pm 0.3 \mathrm{~Hz}, n=31$, $p=0.3988)$. G Quantification of the $\mathrm{XC}\left(\theta, \gamma_{\mathrm{ENV}}\right)$ peak lag in both mouse strains showing the phase relationship between $\theta$ and $\gamma_{\mathrm{ENV}}$ signals ( $p 75^{+/+}$: $-34.8 \pm 6.1 \mathrm{~ms}, n=24$ vs. $\left.p 75^{-1-}:-28.8 \pm 4, n=31 ; p=0.0332\right)$. $\mathbf{H}$ Larger $X C\left(\theta, \gamma_{\mathrm{ENV}}\right)$ peak in $p 75^{-1-}$ mice also indicates a stronger modulation of $\gamma$ amplitude by the underlying $\theta$ rhythms in $p 75^{-1-}$ mice ( $p 75^{+/+}: 0.29 \pm 0.03, n=24 \mathrm{vs}$. $\left.p 75^{-1-}: 0.36 \pm 0.02, n=31 ; p=0.0206\right)$. Data are presented as mean \pm SEM. ${ }^{*} P<0.05$ compared to $p 75^{+/+}$mice.

signal was not significantly different between $\mathrm{p} 75^{+/+}$and p $75^{-1-}$ mice, we tested whether gamma-theta nesting occurs in vitro and whether it was affected by the absence of $\mathrm{p} 75^{\mathrm{NTR}}$ signaling. We proceeded to fit an envelope signal to the peaks of the gamma rhythm, $\gamma_{\mathrm{ENV}}$ (see "Methods," Fig. 2A, B). The spectral analysis of the $\gamma_{\mathrm{ENV}}$ showed that in both mouse strains this waxing and waning oscillation resides within the theta-frequency band (Fig. 2C, D). It was interesting to observe that despite the theta peak frequency of $\gamma_{\mathrm{ENV}}$ remained the same, there was a significant increase in the $\gamma_{\mathrm{ENV}}$ power in the absence of $\mathrm{p} 75^{\mathrm{NTR}}$ signaling (Fig. 2E, F).

To further assess the strength of the nesting, we performed an XC analysis of both signals $\left(\mathrm{XC}\left(\theta, \gamma_{\mathrm{ENV}}\right)\right)$. The $\mathrm{XC}\left(\theta, \gamma_{\mathrm{ENV}}\right)$ displayed a prominent negative peak with a lag close to zero (Fig. 2D), which is indicative of a strong phase locking with the maximum amplitude of gamma oscillations centered at the trough of the theta oscillations $^{37}$ in both mouse strains (Fig. 2A, B, D). However, a difference in the phase relationship between the two signals was evidenced by a significant leftward shift of the $\mathrm{XC}\left(\theta, \gamma_{\mathrm{ENV}}\right)$ in the $\mathrm{p} 75^{+/+}$mice indicating that peaks of $\gamma_{\mathrm{ENV}}$ occur earlier in the theta cycle in p $75^{-1-}$ littermates (Fig. 2D, G) as has been reported to happen in vivo in rat area CA1 of the hippocampus ${ }^{37}$. The magnitude of the $\mathrm{XC}\left(\theta, \gamma_{\mathrm{ENV}}\right)$ peak was also analyzed in order to determine the strength of the theta-gamma coordination. Compared to $\mathrm{p} 75^{+/+}$, $\mathrm{p} 75^{-1-}$ mice showed a significant increase in the $\mathrm{XC}(\theta$, $\left.\gamma_{\mathrm{ENV}}\right)$ peak (Fig. 2D, H), indicating a stronger nesting of gamma within the theta rhythm in the absence of p $75^{\text {NTR }}$ signaling. 
p $75^{-/-}$mice exhibit increased firing synchrony of FSNs during gamma oscillations in the hippocampal network

Cholinergic terminals project to many regions of the hippocampus targeting both CA1 and CA3, where they establish synaptic contacts with a myriad of cell types including GABAergic interneurons. Because of that, tonic cholinergic excitation of interneurons, coupled with their phasic septal GABAergic inhibition, has been suggested to be responsible for the rhythmic discharge of hippocampal interneurons ${ }^{45}$. With the aim to identify the cellular components underlying the observed behavior of neuronal network rhythms and interaction, we performed concomitant recordings of FSN firing and ongoing gamma oscillations induced by $20 \mu \mathrm{M}$ CCh.

Consistent with the higher regularity of gamma oscillations observed in $\mathrm{p} 75^{-1-}$ mice, we found that $\mathrm{p} 75^{-1-}$ FSNs are more entrained into gamma oscillations than FSNs in $\mathrm{p}^{+/+} 5^{+/}$littermates (Fig. 3). This was evidenced by a narrower FSN-AP firing distribution around a gammapreferred phase angle, which resulted in a significantly increased vector length (Fig. 3A-F). It confirms that FSNs fire more synchronized to the gamma rhythm in the absence of $\mathrm{p} 75^{\mathrm{NTR}}$ signaling. However, the preferred phase angle was similar between both mouse strains (Fig. 3G), while the firing rate was lower in $\mathrm{p} 75^{-1-}$ hippocampal CA3 FSNs (Fig. 3H).

In addition to the increase in FSN firing phase locking to the gamma rhythm, we also assessed excitatory postsynaptic currents (EPSCs) in FSNs during ongoing gamma oscillations in order to study the effect of $\mathrm{p} 75^{\mathrm{NTR}}$ signaling loss on the excitatory drive onto this neuronal class (Fig. S5). We found that EPSCs exhibit larger amplitudes in $\mathrm{p} 75^{-/-}$compared to $\mathrm{p} 75^{+/+}$, while no differences were found for EPSC frequency (Fig. S5A-F). Consequently, EPSC charge transfer was higher in $\mathrm{p} 75^{-1-}$ compared to $\mathrm{p} 75^{+/+}$(Fig. S5F).

Interestingly, we observed that EPSC input to FSNs in both mouse strains was also oscillating in the gamma range with a peak frequency in accordance to the frequency of the extracellular gamma oscillation (Fig. S5G, $\mathrm{J})$. To test if there were differences in the rhythmicity in the oscillatory pattern of the EPSC input, we calculated the $\mathrm{Cr}$ and found that $\mathrm{p} 75^{-1-}$-EPSCs displayed a higher rhythmicity compared to p $75^{+/+}$-EPSCs (Fig. S5H, K, M). To further test the relationship between the oscillatory pattern of the excitatory input to FSNs and LFP gamma oscillations, we performed an XC analysis between the filtered EPSC and LFP signals. We found that while XC lags were similar in both mouse strains, the $\mathrm{XC}$ peak showed an increase in $\mathrm{p} 75^{-1-}$ mice (Fig. S5I, L, M). These data are consistent with our previous results suggesting that the ablation of the $\mathrm{p} 75^{\mathrm{NTR}}$ signal increases the synchronicity of the neural network activity in the mouse hippocampus.

\section{Ablation of $p 75^{\mathrm{NTR}}$ signaling prevents $A \beta$-induced degradation of gamma oscillations in the hippocampal network}

It has been observed that $\mathrm{AD}$ patients and $\mathrm{AD}$ mouse models display alterations of cognition-relevant brain rhythm properties and dynamics ${ }^{46-48}$, prior to plaque formation when only small amounts of soluble forms of $\mathrm{A} \beta$ can be detected. We have previously reported that acute $A \beta$ application to hippocampal slices induces a drastic degradation of KA-induced gamma oscillations in area $\mathrm{CA} 3^{31}$. Furthermore, we have characterized in a novel App knock-in AD mouse model ${ }^{49}$ a progressive degradation of gamma oscillations over development, preceding $A \beta$ plaque formation (unpublished data).

Here, we aimed to test whether the increased stability of cognition-relevant neuronal network dynamics caused by p $75^{\text {NTR }}$ ablation could be affected by the presence of $A \beta$. For this purpose, we recorded CCh-induced gamma oscillations from slices preincubated for $15 \mathrm{~min}$ with $50 \mathrm{nM} \mathrm{A} \beta$. Similar to previous findings in WT mice ${ }^{31,33}$, the presence of $A \beta$ led to a significant decrease of gamma oscillation power in $\mathrm{p} 75^{+/+}$mice $(37.8 \%$, Table S1), while the same treatment failed to reduce gamma power in p $75^{-1-}$ littermates (Table S1 and Fig. 4A, B, D). This preventative effect caused by $\mathrm{p} 75^{\mathrm{NTR}}$ ablation was accompanied by prevention of the reduction of gamma oscillation rhythmicity since $A \beta$ had no effect on $\mathrm{Cr}$ in $\mathrm{p} 75^{-/-}$mice, while $\mathrm{p} 75^{+/+}$littermates showed a significant decrease of gamma rhythm quality (Table S1 and Fig. 4B, F). Peak frequency analysis showed that $A \beta$ caused an increase in the principal frequency in the absence of $\mathrm{p} 75^{\mathrm{NTR}}$ signaling, while the peak frequency in p $75^{+/+}$mice remained unaltered (Table S1 and Fig. 1B, E vs. Figure 4B, E). Interestingly, theta oscillation power was significantly reduced in $\mathrm{p} 75^{+/+}$slices pretreated with $\mathrm{A} \beta$ (32.1\% of decrease), while $\mathrm{p} 75^{-1-}$ mice did not show any disruption of the theta rhythm following exposure to $A \beta$ (Table S1 and Fig. 4A, C, D).

\section{Ablation of $p 75^{\text {NTR }}$ signaling prevents $A \beta$-induced disruption of gamma-theta rhythm interaction}

Next, we wondered whether exposure to $A \beta$ could affect the observed strong phase locking of the gamma rhythm to a preferred theta phase. By performing the same analysis described in Figure S4, we found that following $A \beta$ preincubation neither $\mathrm{p} 75^{-/-}$nor $\mathrm{p} 75^{+/+}$showed a significant decrease of the resultant vector length (Table S1 and Fig. S6A-E). However, the preferred phase angle was significantly shifted in $\mathrm{p} 75^{+/+}$mice (Table S1 and Fig. $\mathrm{S} 6 \mathrm{~B}, \mathrm{D}, \mathrm{F})$, indicating that exposure to $A \beta$ led to the occurrence of gamma bouts much earlier in the ascending theta phase in $\mathrm{p} 75^{+/+}$mice. Consistent with previous findings, $A \beta$ exposure induced an increase in the number of gamma peaks/theta cycle in $\mathrm{p} 75^{-1-}$ mice, while this 


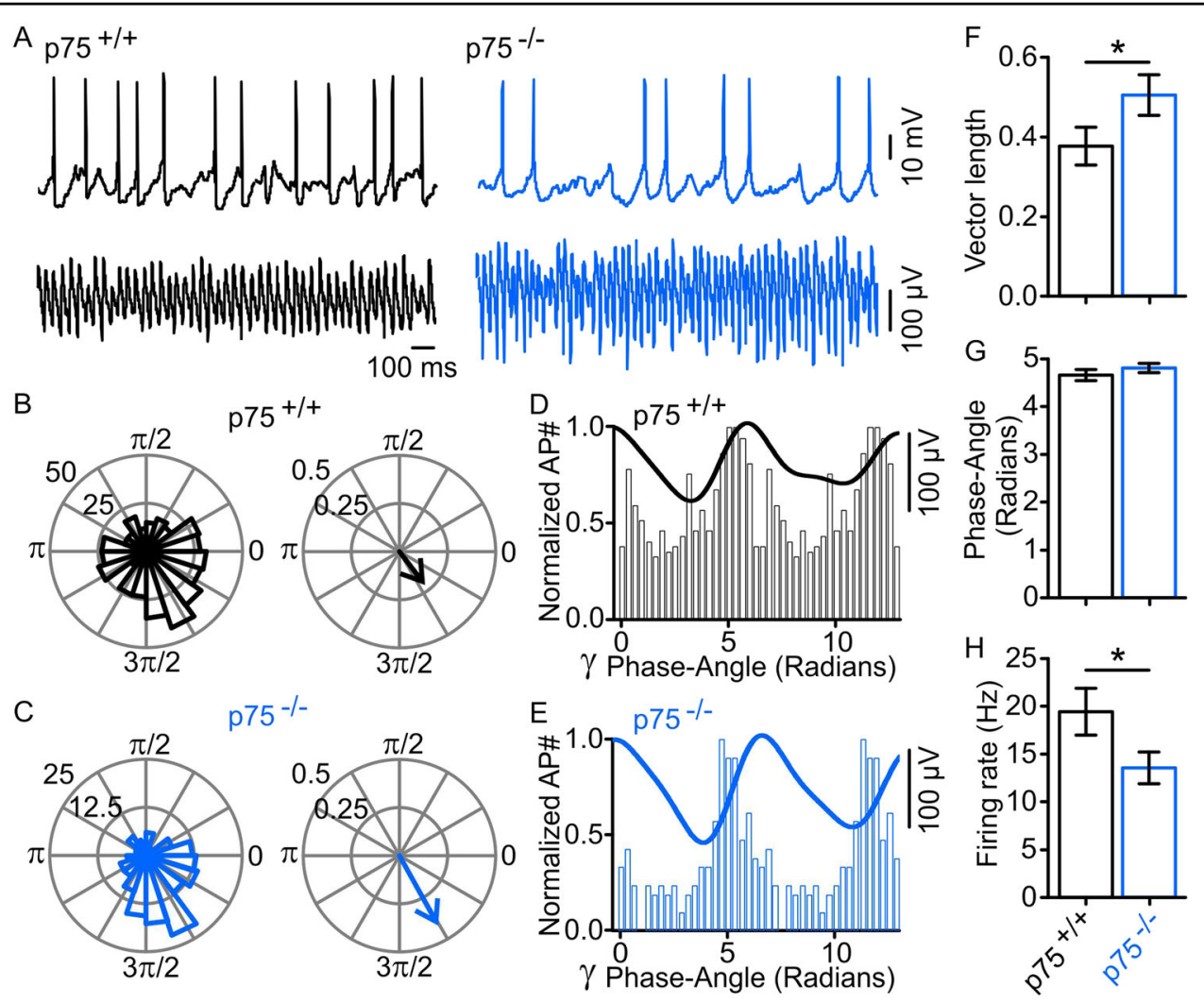

Fig. 3 AP firing window of fast-spiking interneurons in $\mathbf{p} 75^{+/+}$and $\mathbf{p} 75^{-/-}$mice. A Representative traces of AP firing from fast-spiking interneurons were recorded concomitantly with gamma oscillations $(\gamma)$ in area CA3 20 min after $20 \mu \mathrm{M}$ CCh. B Polar plots showing the distribution of APs within the $\gamma$ cycle in $\mathrm{p} 75^{+/+}$mice. Note that a narrower firing distribution resulting in a larger vector in $\mathrm{p} 75^{-1-}$ mice $(\mathbf{C})$ denotes a higher firing synchronization compared to the firing behavior of FSNs in $\mathrm{p} 75^{+/+}$mice. D Normalized AP distribution of two $\gamma$ cycles showing firing coincidence with a representative sample trace from $\mathrm{p} 75^{+/+}$and $\mathrm{p} 75^{-/-}$(E) recordings, also showing a tightened firing window in $\mathrm{p} 75^{-/-}$strain (better AP synchronization). $\mathbf{F}$ Resultant vector length quantification ( $p 75^{+/+}: 0.38 \pm 0.05, n=32 ; p 75^{-1-}: 0.51 \pm 0.05, n=29, p=0.0240$ ). G No significant differences were found in the preferred phase angle between both mouse strains ( $p 75^{+/+}: 4.7 \pm 0.1$ radians, $n=32 ; p 75^{-1-}: 4.8 \pm 0.1$ radians, $n=29$, $p=0.3505)$. H Firing rate quantification $\left(p 75^{+/+}: 19.4 \pm 2.5 \mathrm{~Hz}, n=32 ; p 75^{-/-}: 13.5 \pm 1.7 \mathrm{~Hz}, p=0.0281\right.$ ). Data are presented as mean \pm SEM. ${ }^{*} p<0.05$ compared to $\mathrm{p} 75^{+/+}$mice.

relation remained unaltered in $\mathrm{p} 75^{+/+}$mice (Table S1 and Fig. S6G). Thus, $A \beta$ induced a shift towards faster gamma oscillations in $\mathrm{p} 75^{-1-}$ mice without affecting gamma-theta phase locking or gamma oscillation power and rhythmicity.

Thereafter, we investigated to what extent $A \beta$ might alter the nature of the nesting of both rhythms. For this purpose, we recorded $\mathrm{CCh}$-induced gamma oscillations from hippocampal slices from both $\mathrm{p} 75^{+/+}$and $\mathrm{p} 75^{-/-}$ mice 15 min after preincubation with $A \beta$ and performed $\mathrm{XC}$ and spectral analysis of the envelope signal fitted to gamma peaks. As previously observed, the spectral analysis of $\gamma_{\mathrm{ENV}}$ fitted to gamma peaks showed that in both mouse strains this oscillation resides within the thetafrequency band (Fig. 5A-C). Interestingly, in $\mathrm{p} 75^{+/+}$mice $\mathrm{A} \beta$ caused a slowing of $\gamma_{\mathrm{ENV}}$ compared to $\mathrm{p} 75^{-1-}$, where $\gamma_{\mathrm{ENV}}$ peak frequency remained unaltered (Table S1 and Fig. 5C, F). Notably, in $\mathrm{p} 75^{+/+}$mice $\mathrm{A} \beta$ caused a $45.7 \%$ decrease of $\gamma_{\mathrm{ENV}}$ power, while $\gamma_{\mathrm{ENV}}$ power was not affected by $\mathrm{A} \beta$ in the absence of $\mathrm{p} 75^{\mathrm{NTR}}$ signaling (Table S1 and Fig. 5C, E).

To further elucidate the phase relationship between both $\theta$ and $\gamma_{\mathrm{ENV}}$, we assigned the magnitude and lag of the central peak of the $\mathrm{XC}\left(\mathrm{XC}\left(\theta, \gamma_{\mathrm{ENV}}\right)\right)$ as measurements of the interaction strength and the phase locking of both signals, respectively ${ }^{37}$. Although $A \beta$ did not induce a significant shift in the phase relationship between $\theta$ and $\gamma_{\mathrm{ENV}}$ signals (Table S1 and Fig. 5D, G), the $\mathrm{XC}\left(\theta, \gamma_{\mathrm{ENV}}\right)$ peak of the signals recorded from $\mathrm{p} 75^{+/+}$was significantly decreased by $\mathrm{A} \beta$ exposure, while the $\mathrm{XC}\left(\theta, \gamma_{\mathrm{ENV}}\right)$ peak of the signals recorded from $\mathrm{p} 75^{-1-}$ remained unchanged after $A \beta$ exposure (Table S1 and Fig. 5D, H).

\section{Discussion}

\section{New insights into the role of $p 75^{\text {NTR }}$ signaling in hippocampus physiology}

Although some effects on the survival and proliferation of BFCNs after the ablation of neurotrophin receptors 


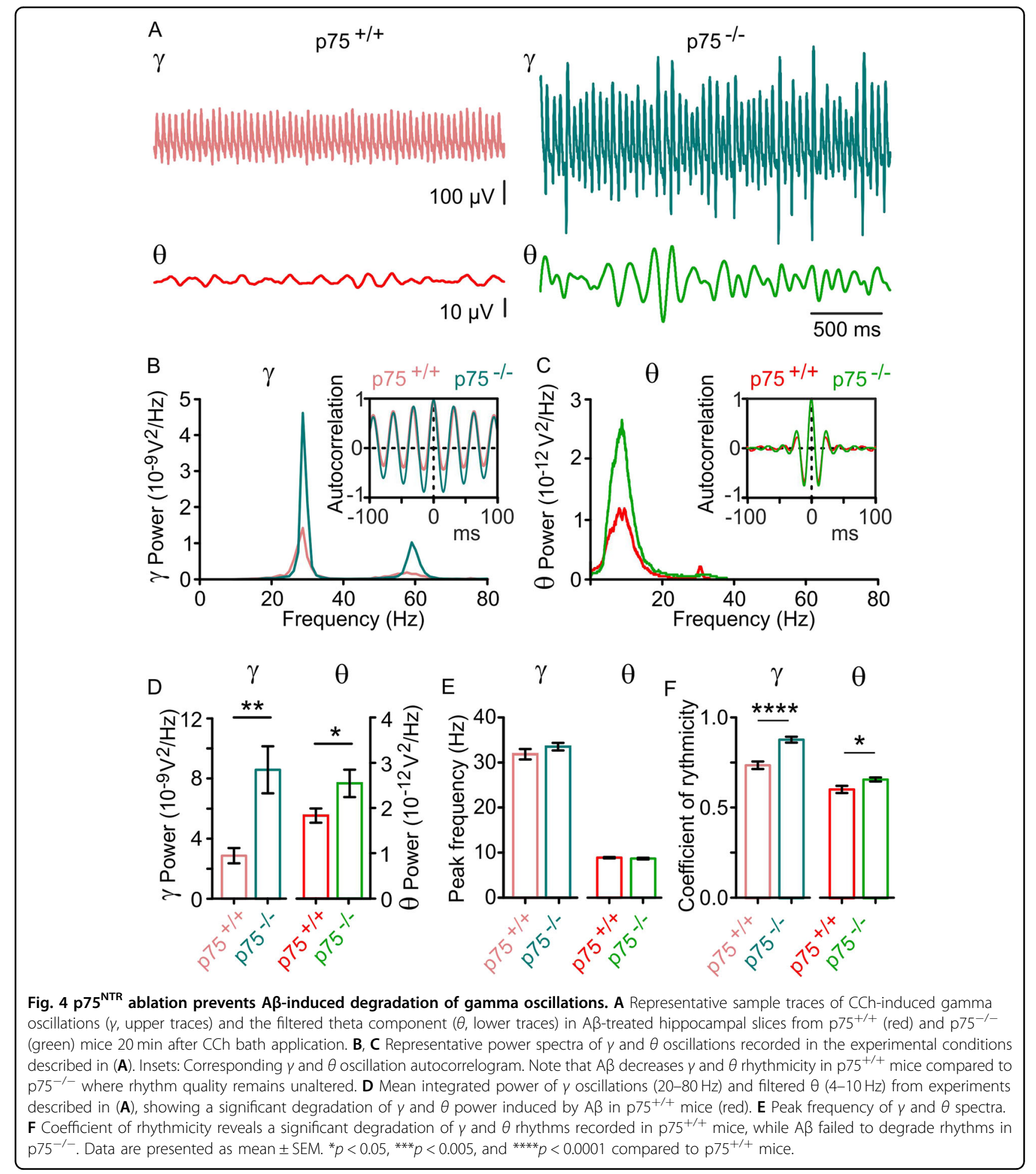

have been described before ${ }^{50-53}$, the consequences of these effects for the cognition-relevant network dynamics of one of the major targets of BFCN output-the hippocampal formation-are not known. Noteworthy, TrkB receptor deficiency in FSNs has been shown to decrease the power of gamma oscillations in the hippocampal network ${ }^{21}$, suggesting that disturbing neurotrophic signaling can have an impact on neuronal network dynamics in the hippocampus.

p $75^{\mathrm{NTR}}$ knockout mice exhibit an increase in cholinergic cell number and size in the basal forebrain, but also an increase in the number of cholinergic axonal fibers 

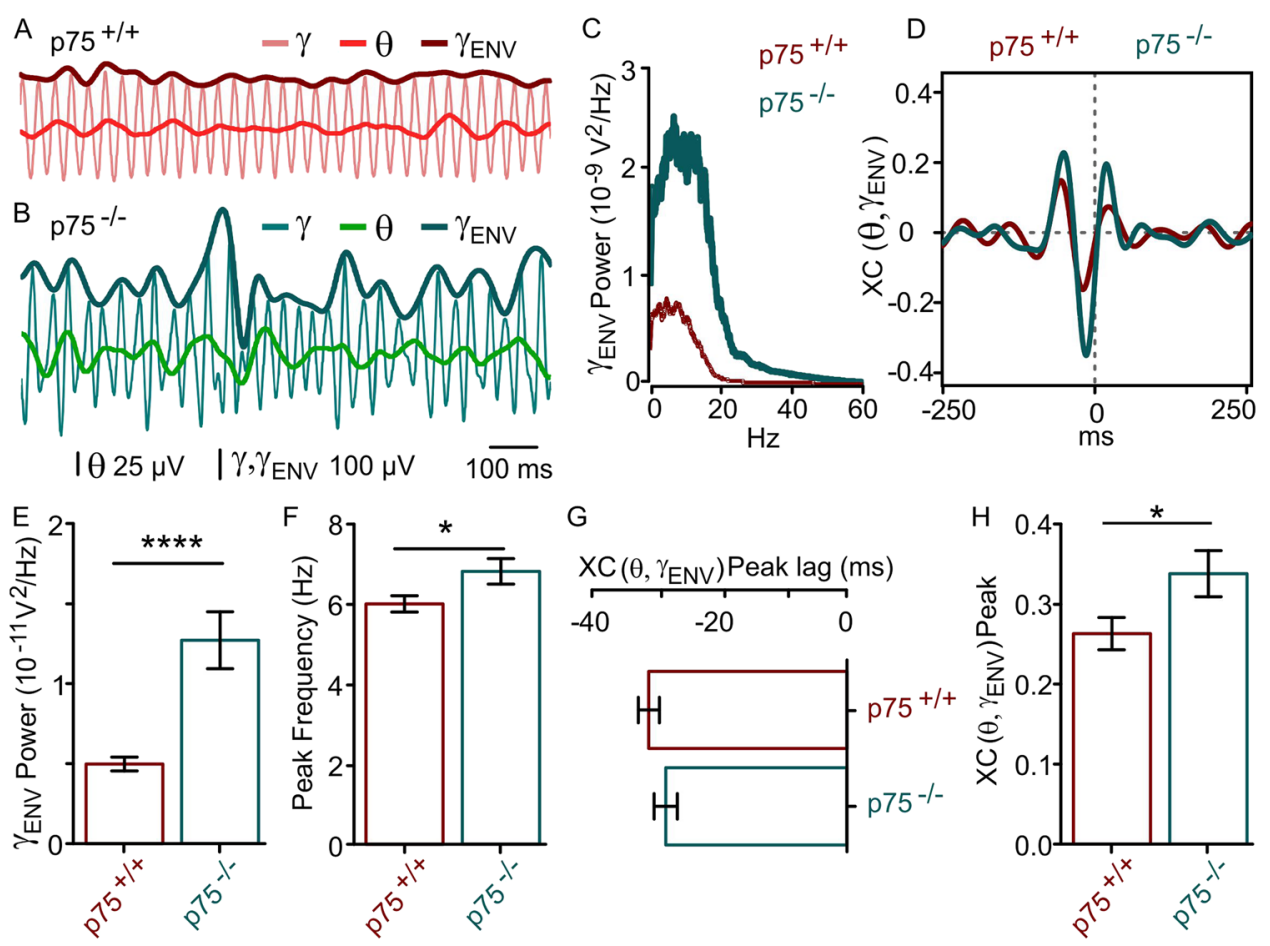

Fig. 5 p $75^{\text {NTR }}$ ablation prevents $A \beta$-induced disruption of gamma-theta nesting. A Representative sample trace of CCh-induced LFPs after A exposure showing a marked reduction of the envelope signal $\gamma_{\text {ENV }}$ (see "Methods"; dark red), $\gamma$ (light red), and $\theta$ (red) signals in p $75^{+/+}$mice. B Representative sample trace of CCh-induced LFPs after A $\beta$ exposure showing no reduction of the envelope signal $\gamma_{\text {ENV }}$ (dark green), $\gamma$ (light green), and $\theta$ (green) signals recorded in $\mathrm{p} 75^{-/-}$mice. C Representative power spectra of the $\gamma_{\mathrm{ENV}}$ signal from $\mathrm{p} 75^{+/+}$(dark red) and $\mathrm{p} 75^{-1-}$ (dark green), showing that $A \beta$ dramatically decreases $\gamma_{\text {ENV }}$ power in $p 75^{+/+}$mice. D Cross-correlation function of $\theta$ with $\gamma_{E N V}\left(X C\left(\theta, \gamma_{E N V}\right)\right)$ obtained from the same data as in $(\mathbf{A}, \mathbf{B})$ showing that $A \beta$ significantly affects the magnitude of the $\theta$ and $\gamma_{\mathrm{ENV}}$ cross-correlation. $\mathbf{E}$ Mean $\gamma_{\mathrm{ENV}}$ power within the $\theta$ frequency band shows that $A \beta$ drastically decreases $\gamma$ amplitude modulation of $p 75^{+/+} \gamma$ oscillations, while $\gamma_{\mathrm{ENV}}$ power of $p 75^{-/-}$mice remains unaltered. F Peak frequency of the spectra as in $(\mathbf{C})$ from all slices recorded showing that the $\gamma$ amplitude modulation is oscillating within the $\theta$ range in both $\mathrm{p} 75^{+/+}$and $\mathrm{p} 75^{-/-}$mice. G Quantification of the $X C\left(\theta, \gamma_{\mathrm{ENV}}\right)$ peak lag in both mouse strains showing the phase relationship between $\theta$ and $\gamma_{\mathrm{ENV}}$ signals. $\mathbf{H}$ The larger $X C\left(\theta_{1}, \gamma_{E N V}\right)$ peak in $p 75^{-1-}$ mice signifies a stronger modulation of $\gamma$ amplitude by the underlying $\theta$ rhythm, indicating that p $75^{\mathrm{NTR}}$ ablation prevents the A $\beta$-induced impairment of $\theta-\gamma$ nesting observed in $p 75^{+/+}$mice. Data are presented as mean \pm SEM. ${ }^{*} P<0.05$ and **** $p<0.0001$ compared to $p 75^{+/+}$mice.

targeting the hippocampus, as well as an increased choline acetyltransferase activity ${ }^{50,54}$. In the present study, we report for the first time that this increase of cholinergic input onto the hippocampus has a cognition-relevant physiological consequence. We describe a marked increase in the power of CCh-induced gamma oscillations in area CA3 hippocampal of $\mathrm{p} 75^{-1-}$ mice, which is consistent with histochemical studies. In addition to the increased gamma oscillation power, gamma activity was also more rhythmic in $\mathrm{p} 75^{-1-}$ mice, most likely caused by the observed increased synchrony of FSN activity. A further physiological consequence is the stronger interaction between gamma and theta oscillators, which was evidenced by an increased phase lock of the gamma rhythm to the underlying theta rhythm. Our functional findings support the idea that $\mathrm{p} 75^{\mathrm{NTR}}$ plays a key role in the pruning of hippocampal cholinergic innervation, at the very least in the early stages of development ${ }^{25,55}$, and has a strong impact on cognition-relevant neuronal network dynamic in the hippocampus. Our data provide the basis for further studies into the nature of these rhythms interaction in vitro as well as in vivo in the absence of p $75^{\mathrm{NTR}}$ signaling.

\section{Ablation of $\mathrm{p} 75^{\mathrm{NTR}}$ signaling increases gamma-theta rhythm coupling}

Theta oscillations have been reported to interact with and change the properties of the gamma rhythm, such as amplitude, phase, or power of the oscillation ${ }^{4-6,8,11,12,56}$. Although other studies have reported large theta oscillations in vitro ${ }^{57-59}$, in our brain slice preparations theta oscillations were more modest. This is very likely due to fundamental differences in the experimental procedures such as the cutting orientation of the slices (horizontal rather than transverse to generate robust gamma oscillations) or the fact that some studies use cocktails of receptor agonists and antagonists to elicit theta oscillations ${ }^{57}$, while others use whole hippocampal preparations to study spontaneously generated theta rhythms ${ }^{58}$. 
Nonetheless, even though our experiments were designed to favor the recording of gamma oscillations, we were able to obtain a small but consistent underlying theta rhythm that could be isolated by filtering the LFP signal in order to remove the dominant gamma oscillations (see theta $\mathrm{Cr}$ values in Figs. 1 and 5). This allowed us to study the distribution of gamma peaks to the instantaneous theta phase as well as the nesting of gamma in the underlying theta rhythms. To our knowledge, our study is the first to show that CCh application $(20 \mu \mathrm{M})$ can induce gamma and theta rhythms that coexist and are phaselocked in area CA3 of an acute hippocampal slice preparation. Therefore, our results add relevant evidence to the notion that an intrinsic theta oscillator exists in the hippocampus ${ }^{16,60}$.

In our study, the spectral analysis of LFP gamma oscillations in $\mathrm{p} 75^{-1-}$ mouse networks showed a leftward shift of the peak frequency, which suggested that there is a slowing of the gamma rhythm in the absence of $\mathrm{p} 75^{\mathrm{NTR}}$ signaling. Indeed, gamma autocorrelograms and gamma peaks/theta cycle analysis (see Fig. S4) corroborated this slowing phenomenon. Notably, the preferred theta phase angle was different for each of the two mouse strains. Altogether, these factors may effectively alter the phase-phase interaction of theta and gamma oscillations. In fact, the distribution analysis of gamma peaks within a theta cycle showed a stronger preference (locking) of gamma to the preferred phase angle of the underlying theta wave when $\mathrm{p} 75^{\mathrm{NTR}}$ signaling is absent (see Fig. S4). Given that no significant spectral differences of the theta rhythm were found between both mouse strains, it seems unlikely that the theta oscillation was the main driver of the increased phase locking. Instead, our data suggest that this stronger gamma-phase coupling arises from alterations of the cellular mechanisms that control the dynamics of hippocampal rhythms.

It has been proposed that such an underlying cellular mechanism could be the enhancement of the precision of AP firing timing within a slow oscillation (i.e., theta) cycle by fast precisely tuned hyperpolarizations of the membrane potential ${ }^{61}$. This principle appears to be particularly relevant in hippocampal local circuits where interneurons, especially FSNs, play a central role in determining the firing rate and phase of pyramidal cells during ongoing gamma oscillations ${ }^{62}$. This proper timing is critical for maintaining network stability. In fact, activation of FSNs powerfully controls the PC network and rhythm generation optimally at $8 \mathrm{~Hz}$, while silencing them disrupts an intrinsic theta rhythm in $\mathrm{CA}^{63}$. Interestingly, on the cellular level, we found that FSNs from $\mathrm{p} 75^{-1-}$ mice displayed a more synchronized activity during the gamma rhythm compared to $\mathrm{p} 75^{+/+}$FSN firing activity, and with no difference in the gamma-preferred $\mathrm{p}$ hase angle. This better synchronized activity of FSNs likely accounts for the differences observed between both mouse strains since a neural network more entrained to the gamma rhythm will be also more attuned to the underlying theta rhythm as is the case in $\mathrm{p} 75^{-1-}$ hippocampus with better synchronized FSN activity. On the other hand, a larger and more gamma-locked excitatory input to FSNs has been observed in $\mathrm{p} 75^{-1-}$ mice, while an overall decrease of excitability was apparent. The most plausible interpretation of these findings may be found in an enhancement of the postsynaptic computational capacity of FSNs to respond to glutamatergic inputs from pyramidal neurons in a situation where the mechanism of excitatory event integration is more synchronized (see Fig. S5).

In addition, we also tested whether the theta-attuned gamma oscillations (here induced by $\mathrm{CCh}$ ) also exhibit an oscillating amplitude peak patterned in the theta band as it occurs in vivo ${ }^{37}$. Our data showed gamma-theta nesting in both mouse strains with better coupling of the rhythms in the absence of $\mathrm{p} 75^{-1-}$ signaling. Moreover, $\mathrm{p} 75^{-1-}$ mice displayed a larger-amplitude oscillation. This opens the possibility for the hippocampal microcircuitry to resonate without a larger external theta input, which could lead to an increase in gamma oscillation power via a mechanism similar to the one operating in the intact brain. Studies directed to dissect the modulation phenomena will further our understanding of the theta-gamma interactions observed here. It is known that the inputs from the GABAergic inhibitory neurons from the MS/DBB-regarded as the main theta input into the hippocampus-can greatly influence this modulation $^{16,17,60,64}$. In line with the better synchronization with the gamma rhythm observed for the FSN firing behavior in $\mathrm{p} 75^{-1-}$ mice, it has been proposed that the amplitude modulation of the gamma rhythm at theta frequencies may arise from a phasic interruption of the interneuronal network activity, as the gamma power and interneuronal firing are both modulated at theta and are in phase with each other ${ }^{1}$.

Taken together, and consistent with previous reports ${ }^{17,65,66}$, both gamma-theta phase locking/nesting and gamma oscillation strengthening in the presence of CCh strongly support the notion that the role of the cholinergic innervation of the hippocampal regionincreased due to $\mathrm{p} 75^{\mathrm{NTR}}$ ablation-is to facilitate the coordination between the gamma rhythms generated in the hippocampus and GABAergic-driven theta rhythms generated also in the MS/DBB.

\section{Functional implications of $\mathrm{p} 75^{\mathrm{NTR}}$ signaling modulation for AD}

Normal neuronal synchrony underlies the generation of brain rhythms, such as gamma oscillations, that promote cognitive functions. There is increasing evidence that the disruption of these rhythms can be used as an early 
functional biomarker of $\mathrm{AD}$ since initial alterations in hippocampal network activity arise long before $A \beta$ accumulation and plaque formation. Both disruption of brain rhythms and aberrant $A \beta$ accumulation constitute the pathophysiological and histological hallmarks of the disease, respectively. Moreover, these disease features correlate with the progressive cognitive decline patients experience with the advance of AD to ever more severe stages. Supporting evidence comes from animal studies in which robust alterations of theta-gamma coupling in area CA1/subiculum of AD mouse models was observed before $A \beta$ overproduction ${ }^{47,67}$.

We have also observed that acute exposure of neuronal networks to $A \beta$ induces firing desynchronization and degradation of hippocampal gamma oscillations in vitro ${ }^{31,33,36}$. Consistent with previous findings, here we have observed that acute $A \beta$ induced degradation of gamma oscillations and rhythmicity in $\mathrm{p} 75^{+/+}$mice. Theta oscillation power was also decreased, and a significant disruption of theta-gamma nesting was evident in area CA3 of $\mathrm{p} 75^{+/+}$hippocampal slices. It is noteworthy that the rhythmicity of theta oscillations also significantly decreased compared to $\mathrm{p} 75^{-1-} \mathrm{A} \beta$-treated slices. Given the drastic disruption of both rhythms and their interaction, it is tempting to speculate that the $\mathrm{A} \beta$-induced shift of the gamma-preferred theta phase angle (see Fig. S6 and Table S1) may be responsible for the observed gamma rhythm slowing and decrease of the faster and larger gamma bouts within the theta range when $\mathrm{p} 75^{\mathrm{NTR}}$ signaling is intact.

p $75^{\text {NTR }}$ promotes $A \beta$ neuritic dystrophy both in vivo and in vitro ${ }^{28}$, and is upregulated in the hippocampus of late-stage $\mathrm{AD}$ patients ${ }^{68}$. This upregulation of $\mathrm{p} 75^{\mathrm{NTR}}$ expression in $\mathrm{AD}$ patients' brain parallels the one observed in AD mouse models ${ }^{69}$. Synaptic loss is one of the features of neuronal damage in $\mathrm{AD}$, and it strongly correlates with cognitive impairment in patients ${ }^{70,71}$. Interestingly, the presence of interfering molecules that potentially prevent $\mathrm{A} \beta-\mathrm{p} 75^{\mathrm{NTR}}$ interaction has been shown to have neuroprotective properties ${ }^{69,72}$. Moreover, age-dependent degeneration of BCNF is prevented by modulation of $\mathrm{p} 75^{\mathrm{NTR}} 19$.

Highly complementary to these previous studies at cellular and molecular levels, our functional findings reveal that $\mathrm{p} 75^{\mathrm{NTR}}$ ablation significantly strengthens and protects neuronal network dynamics from $A \beta$-induced neuronal network dysfunction. The $\mathrm{p} 75^{-1-}$ mouse strain used in our study lacks the extracellular receptor domain capable of interacting with extracellular ligands. As such, it displayed a gamma rhythm with increased power and increased rhythmicity, as well as better synchronized FSN activity. The latter appears to be the profound functional effect caused by the deletion of $\mathrm{p} 75^{\mathrm{NTR}}$ signaling since a more synchronized FSN activity leads to the rescue of gamma oscillations during sharp waves ripples, decreases $A \beta$ load, and induces a turnover of microglia from a proinflammatory phenotype towards a phagocytic state in the $5 X F A D$ AD mouse model in vivo ${ }^{48}$. In addition, optogenetically driving medial septal FSNs restores hippocampal slow gamma oscillation amplitude as well as phase-amplitude coupling of the J20 AD mouse model ${ }^{73}$. Finally, our study identifies $\mathrm{p} 75^{\mathrm{NTR}}$ as a suitable target in the search for neuroprotective and/or neurorestorative strategies against $\mathrm{AD}$ that opens treatment avenues for other cognitive-compromised and amyloid-driven pathologies. Studies that include the blockade of $\mathrm{p} 75^{\mathrm{NTR}}$ signaling as a therapeutic step during AD progression might help to counteract the degeneration of cholinergic neurons, altered neuronal network dynamics, and, hence, the cognitive dysfunction typical of AD.

\section{Acknowledgements}

This work was supported by the Swedish Research Council, the Swedish Brain Foundation and the Swedish Alzheimer Foundation (A.F.), the Loo and Hans Ostermans Foundation and Foundation for Geriatric Diseases at Karolinska Institutet (Y.A.-T.), and a CONACYT postdoctoral fellowship (H.B.-T.).

\section{Author contributions}

Y.A.-T., H.B.-T., and P.D.-G. performed experiments and analyzed data. G.C. and J.J. provided A 42 protein. A.F. designed, funded, and supervised the study. Y.A.-T. and A.F. wrote the manuscript. All authors read and approved the final manuscript.

\section{Funding}

Open access funding provided by Karolinska Institute.

Conflict of interest

The authors declare no competing interests.

\section{Publisher's note}

Springer Nature remains neutral with regard to jurisdictional claims in published maps and institutional affiliations.

Supplementary information The online version contains supplementary material available at https://doi.org/10.1038/s41398-021-01332-8.

Received: 7 December 2020 Revised: 9 March 2021 Accepted: 26 March 2021

Published online: 09 April 2021

\footnotetext{
References

1. Buzsáki, G. et al. Hippocampal network patterns of activity in the mouse. Neuroscience 116, 201-211 (2003).

2. Colgin, L. L. Theta-gamma coupling in the entorhinal-hippocampal system. Curr. Opin. Neurobiol. 31, 45-50 (2015).

3. Whittington, M. A. \& Traub, R. D. Interneuron diversity series: inhibitory interneurons and network oscillations in vitro. Trends Neurosci. 26, 676-682 (2003).

4. Bragin, A. et al. Gamma (40-100 Hz) oscillation in the hippocampus of the behaving rat. J. Neurosci. 15, 47-60 (1995).

5. Buzsàki, G. \& Eidelberg, E. Phase relations of hippocampal projection cells and interneurons to theta activity in the anesthetized rat. Brain Res. 266, 334-339 (1983).

6. Canolty, R. T. et al. High gamma power is phase-locked to theta oscillations in human neocortex. Science 313, 1626-1628 (2006).

7. Demiralp, T. et al. Gamma amplitudes are coupled to theta phase in human EEG during visual perception. Int. J. Psychophysiol. 64, 24-30 (2007).
} 
8. Mormann, F. et al. Phase/amplitude reset and theta-gamma interaction in the human medial temporal lobe during a continuous word recognition memory task. Hippocampus 15, 890-900 (2005).

9. Sauseng, P. et al. Brain oscillatory substrates of visual short-term memory capacity. Curr. Biol. 19, 1846-1852 (2009).

10. Dürschmid, S. et al. Phase-amplitude cross-frequency coupling in the human nucleus accumbens tracks action monitoring during cognitive control. Front. Hum. Neurosci. 7, 635 (2013).

11. Belluscio, M. A., Mizuseki, K., Schmidt, R., Kempter, R. \& Buzsáki, G. Crossfrequency phase-phase coupling between $\theta$ and $\gamma$ oscillations in the hippocampus. J. Neurosci. 32, 423-435 (2012).

12. Butler, J. L., Mendonça, P. R. F., Robinson, H. P. C. \& Paulsen, O. Intrinsic cornu ammonis area 1 theta-nested gamma oscillations induced by optogenetic theta frequency stimulation. J. Neurosci. 36, 4155-4169 (2016).

13. Lisman, J. E. \& Jensen, O. The theta-gamma neural code. Neuron 77, 1002-1016 (2013).

14. Buzsáki, G. \& Draguhn, A. Neuronal oscillations in cortical networks. Science 304, 1926-1929 (2004)

15. Pastoll, H., Solanka, L., van Rossum, M. C. W. \& Nolan, M. F. Feedback inhibition enables theta-nested gamma oscillations and grid firing fields. Neuron 77, 141-154 (2013).

16. Buzsáki, G. Theta oscillations in the hippocampus. Neuron 33, 325-340 (2002)

17. Lee, M. G., Chrobak, J. J., Sik, A., Wiley, R. G. \& Buzsáki, G. Hippocampal theta activity following selective lesion of the septal cholinergic system. Neuroscience 62, 1033-1047 (1994).

18. Salehi, A., Ocampo, M., Verhaagen, J. \& Swaab, D. F. P75 neurotrophin receptor in the nucleus basalis of Meynert in relation to age, sex, and Alzheimer's disease. Exp. Neurol. 161, 245-258 (2000).

19. Xie, Y., Meeker, R. B., Massa, S. M. \& Longo, F. M. Modulation of the p75 neurotrophin receptor suppresses age-related basal forebrain cholinergic neuron degeneration. Sci. Rep. 9, 5273 (2019).

20. Härtig, W. et al. Functional recovery of cholinergic basal forebrain neurons under disease conditions: old problems, new solutions? Rev. Neurosci. 13, 95-165 (2002).

21. Zheng, $K$. et al. TrkB signaling in parvalbumin-positive interneurons is critical for gamma-band network synchronization in hippocampus. Proc. Natl Acad. Sci. USA 108, 17201-17206 (2011).

22. Hefti, F. Nerve growth factor promotes survival of septal cholinergic neurons after fimbrial transections. J. Neurosci. 6, 2155-2162 (1986).

23. Vantini, G. et al. Evidence for a physiological role of nerve growth factor in the central nervous system of neonatal rats. Neuron 3, 267-273 (1989).

24. Lucidi-Phillipi, C. A., Clary, D. O., Reichardt, L. F. \& Gage, F. H. TrkA activation is sufficient to rescue axotomized cholinergic neurons. Neuron 16,653-663 (1996).

25. Coulson, E. J., May, L. M., Sykes, A. M. \& Hamlin, A. S. The role of the p75 neurotrophin receptor in cholinergic dysfunction in Alzheimer's disease. Neuroscientist 15, 317-323 (2009).

26. Van Der Zee, C. E. E. M., Ross, G. M., Riopelle, R. J. \& Hagg, T. Survival of cholinergic forebrain neurons in developing p75(NGFR)- deficient mice. Science 274, 1729-1732 (1996).

27. Zagrebelsky, $\mathrm{M}$. The $\mathrm{p} 75$ neurotrophin receptor negatively modulates dendrite complexity and spine density in hippocampal neurons. J. Neurosci. 25, 9989-9999 (2005).

28. Knowles, J. K. et al. The p75 neurotrophin receptor promotes amyloid- (1-42)induced neuritic dystrophy in vitro and in vivo. J. Neurosci. 29, 10627-10637 (2009).

29. Ovsepian, S. V. et al. Neurotrophin receptor p75 mediates the uptake of the amyloid beta $(A \beta)$ peptide, guiding it to lysosomes for degradation in basal forebrain cholinergic neurons. Brain Struct. Funct. 219, 1527-1541 (2014).

30. Leão, R. N., Colom, L. V., Borgius, L., Kiehn, O. \& Fisahn, A. Medial septal dysfunction by $A \beta$-induced KCNQ channel-block in glutamatergic neurons. Neurobiol. Aging 33, 2046-2061 (2012).

31. Kurudenkandy, F. R. R. et al. Amyloid-induced action potential desynchronization and degradation of hippocampal gamma oscillations is prevented by interference with peptide conformation change and aggregation. J. Neurosci. 34, 11416-11425 (2014).

32. Andersson, R., Johnston, A. \& Fisahn, A. Dopamine D4 receptor activation increases hippocampal gamma oscillations by enhancing synchronization of fast-spiking interneurons. PLOS ONE 7, https://doi.org/10.1371/journal. pone.0040906 (2012).
33. Andrade-Talavera, Y., Arroyo-García, L. E., Chen, G., Johansson, J. \& Fisahn, A. Modulation of Kv3.1/Kv3.2 promotes gamma oscillations by rescuing $A \beta$-induced desynchronization of fast-spiking interneuron firing in an $A D$ mouse model in vitro. J. Physiol. 598, https://doi.org/10.1113/JP279718 (2020).

34. Fisahn, A., Pike, F. G., Buhl, E. H. \& Paulsen, O. Cholinergic induction of network oscillations at $40 \mathrm{~Hz}$ in the hippocampus in vitro. Nature 394, 186-189 (1998).

35. Andersson, R., Lindskog, M. \& Fisahn, A. Histamine $\mathrm{H3}$ receptor activation decreases kainate-induced hippocampal gamma oscillations in vitro by action potential desynchronization in pyramidal neurons. J. Physiol. 588, 1241-1249 (2010).

36. Balleza-Tapia, H. et al. TrpV1 receptor activation rescues neuronal function and network gamma oscillations from $A \beta$-induced impairment in mouse hippocampus in vitro. Elife 7, 1-24 (2018).

37. Hentschke, H., Perkins, M. G., Pearce, R. A. \& Banks, M. I. Muscarinic blockade weakens interaction of gamma with theta rhythms in mouse hippocampus. Eur. J. Neurosci. 26, 1642-1656 (2007).

38. Chen, G. et al. Bri2 BRICHOS client specificity and chaperone activity are governed by assembly state. Nat. Commun. 8, https://doi.org/10.1038/s41467017-02056-4 (2017).

39. Cohen, S. I. A. et al. A molecular chaperone breaks the catalytic cycle that generates toxic Aß oligomers. Nat. Struct. Mol. Biol. 22, 207-213 (2015).

40. Chen, $\mathrm{G}$. et al. Augmentation of Bri2 molecular chaperone activity against amyloid- $\beta$ reduces neurotoxicity in mouse hippocampus in vitro. Commun. Biol. 3, 32 (2020).

41. Heynen, A. J. \& Bilkey, D. K. Induction of RSA-like oscillations in both the in-vitro and in-vivo hippocampus. Neuroreport 2, 401-404 (1991).

42. Monmaur, P., Ayadi, K. \& Breton, P. Hippocampal EEG responses induced by carbachol and atropine infusions into the septum and the hippocampus in the urethane-anaesthetized rat. Brain Res. 631, 317-324 (1993).

43. Buhl, E. H., Tamás, G. \& Fisahn, A. Cholinergic activation and tonic excitation induce persistent gamma oscillations in mouse somatosensory cortex in vitro. J. Physiol. 513, 117-126 (1998).

44. Traub, R. D. et al. A model of gamma-frequency network oscillations induced in the rat CA3 region by carbachol in vitro. Eur. J. Neurosci. 12, 4093-4106 (2000).

45. Stewart, M. \& Fox, S. E. Firing relations of lateral septal neurons to the hippocampal theta rhythm in urethane anesthetized rats. Exp. Brain Res. 79, 92-96 (1990).

46. Palop, J. J. \& Mucke, L. Network abnormalities and interneuron dysfunction in Alzheimer disease. Nat. Rev. Neurosci. 17, 777-792 (2016).

47. Goutagny, R. et al. Alterations in hippocampal network oscillations and thetagamma coupling arise before $A \beta$ overproduction in a mouse model of Alzheimer's disease. Eur. J. Neurosci. 37, 1896-1902 (2013).

48. laccarino, H. F. et al. Gamma frequency entrainment attenuates amyloid load and modifies microglia. Nature 540, 230-235 (2016).

49. Saito, T. et al. Single App knock-in mouse models of Alzheimer's disease. Nat Neurosci. 17, 661-663 (2014).

50. Yeo, T. T. et al. Absence of p75NTR causes increased basal forebrain cholinergic neuron size, choline acetyltransferase activity, and target innervation. J. Neurosci. 17, 7594-7605 (1997).

51. Naumann, T. et al. Complete deletion of the neurotrophin receptor p75NTR leads to long-lasting increases in the number of basal forebrain cholinergic neurons. J. Neurosci. 22, 2409-2418 (2002).

52. Greferath, U. et al. Enlarged cholinergic forebrain neurons and improved spatial learning in p75 knockout mice. Eur. J. Neurosci. 12, 885-893 (2000).

53. Boskovic, Z. et al. The role of p75NTR in cholinergic basal forebrain structure and function. J. Neurosci. 34, 13033-13038 (2014).

54. Greferath, U., Trieu, J. \& Barrett, G. L. The p75 neurotrophin receptor has nonapoptotic antineurotrophic actions in the basal forebrain. J. Neurosci. Res. 90, 278-287 (2012)

55. Singh, K. K. et al. Developmental axon pruning mediated by BDNF-p75NTRdependent axon degeneration. Nat. Neurosci. 11, 649-658 (2008).

56. Soltesz, I. \& Deschênes, M. Low- and high-frequency membrane potential oscillations during theta activity in CA1 and CA3 pyramidal neurons of the rat hippocampus under ketamine-xylazine anesthesia. J. Neurophysiol. 70, 97-116 (1993).

57. Konopacki, J. \& Gołębiewski, H. Theta-like activity in hippocampal formation slices: cholinergic-gabaergic interaction. Neuroreport 4, 963-966 (1993).

58. Goutagny, R., Jackson, J. \& Williams, S. Self-generated theta oscillations in the hippocampus. Nat. Neurosci. 12, 1491-1493 (2009). 
59. MacVicar, B. A. \& Tse, F. W. Local neuronal circuitry underlying cholinergic rhythmical slow activity in CA3 area of rat hippocampal slices. J. Physiol. 417 197-212 (1989).

60. Colgin, L. L. Mechanisms and functions of theta rhythms. Annu. Rev. Neurosci. 36, 295-312 (2013).

61. Schaefer, A. T., Angelo, K., Spors, H. \& Margrie, T. W. Neuronal oscillations enhance stimulus discrimination by ensuring action potential precision. PLoS Biol. 4, 1010-1024 (2006).

62. Tikidji-Hamburyan, R. A., Martínez, J. J., White, J. A. \& Canavier, C. C. Resonant interneurons can increase robustness of gamma oscillations. J. Neurosci. 35, 15682-15695 (2015)

63. Amilhon, B. et al. Parvalbumin interneurons of hippocampus tune population activity at theta frequency. Neuron $\mathbf{8 6}, 1277-1289$ (2015).

64. Manseau, F., Goutagny, R., Danik, M. \& Williams, S. The hippocamposeptal pathway generates rhythmic firing of GABAergic neurons in the medial septum and diagonal bands: an investigation using a complete septohippocampal preparation in vitro. J. Neurosci. 28, 4096-4107 (2008).

65. Bassant, M. H., Apartis, E., Jazat-Poindessous, F. R., Wiley, R. G. \& Lamour, Y. A. Selective immunolesion of the basal forebrain cholinergic neurons: effects on hippocampal activity during sleep and wakefulness in the rat. Neurodegeneration 4, 61-70 (1995).
66. Apartis, E., Poindessous-Jazat, F. R., Lamour, Y. A. \& Bassant, M. H. Loss of rhythmically bursting neurons in rat medial septum following selective lesion of septohippocampal cholinergic system. J. Neurophysiol. 79, 1633-1642 (1998).

67. Mondragón-Rodríguez, S., Gu, N., Manseau, F. \& Williams, S. Alzheimer's transgenic model is characterized by very early brain network alterations and $\beta$-CTF fragment accumulation: reversal by $\beta$-secretase inhibition. Front. Cell Neurosci. 12, 1-17 (2018).

68. Chakravarthy, B. et al. Hippocampal membrane-associated p75ntr levels are increased in Alzheimer's disease. J. Alzheimer's Dis. 30, 675-684 (2012).

69. Yao, X. Q. et al. P75NTR ectodomain is a physiological neuroprotective molecule against amyloid-beta toxicity in the brain of Alzheimer's disease. Mol. Psychiatry 20, 1301-1310 (2015).

70. Katzman, R. et al. Physical basis of cognitive alterations in alzheimer's disease: Synapse loss is the major correlate of cognitive impairment. Ann. Neurol. $\mathbf{3 0}$ 572-580 (2005)

71. Coleman, P. D. \& Yao, P. J. Synaptic slaughter in Alzheimer's disease. Neurobiol. Aging 24, 1023-1027 (2003).

72. Yang, T. et al. Small molecule, non-peptide p75NTR ligands inhibit Aß-induced neurodegeneration and synaptic impairment. PLoS ONE 3, e3604 (2008).

73. Etter, G. et al. Optogenetic gamma stimulation rescues memory impairments in an Alzheimer's disease mouse model. Nat. Commun. 10, 5322 (2019). 